\title{
Forming nanomaterials as layered functional structures toward materials nanoarchitectonics
}

\begin{abstract}
Katsuhiko Ariga ${ }^{1,2}$, Qingmin $\mathrm{Ji}^{1}$, Jonathan $\mathrm{P}$ Hill ${ }^{1,2}$, Yoshio Bando ${ }^{1}$ and Masakazu Aono ${ }^{1}$
Although progress in nanotechnology is anticipated to stimulate the development of innovative materials, the areas of nanotechnology and materials science are somehow separated with little common ground in current technologies. Nanotechnology has some analytical aspects that are beneficial mainly to the nanoscopic sciences at the atom or molecular levels. Experience of these advanced techniques has only been applied in synthetic approaches to macroscopic materials in rather immature level. Forming nanomaterials into hierarchic and organized structures is a rational way of preparing advanced functional materials. Recently, one of us coined the term materials' nanoarchitectonics to express this innovation. This review focuses on recent research to develop functional materials by forming nanomaterials into organized structures, especially in well-ordered layered structural motifs. This layered nanoarchitectonics can be achieved by using the versatile technology of layer-by-layer assembly. Reassembly of bulk materials into novel layered structures through layered nanoarchitectonics has created many innovative functional materials in a wide variety of fields as can be seen in ferromagnetic nanosheets, sensors, flame-retardant coatings, transparent conductors, electrodes and transistors, walking devices, drug release surfaces, targeting drug carriers and cell culturing.
\end{abstract}

NPG Asia Materials (2012) 4, e17; doi:10.1038/am.2012.30; published online 18 May 2012

Keywords: architectonics; nanomaterials; layers; layer-by-layer assembly; device; sensor; drug delivery

\section{INTRODUCTION: NANOARCHITECTONICS FOR MATERIALS INNOVATION}

Rapid progress in nanotechnology is anticipated to stimulate the development of innovative materials with advanced functions. In addition to the currently available compact high-speed computing devices, other extremely efficient solar energy convertors and selfdetermining drug delivery systems based on functional atomic or molecular materials would have great societal impacts. The nanotech and materials' worlds are apparently separated with little common ground in current technology. Nanotechnology has some analytical aspects that are beneficial to the nanoscopic sciences at the atomic or molecular levels. Experience of these advanced techniques has only been applied in synthetic approaches to macroscopic materials at a rather immature level. Preparation of functional materials based fully on atom/molecular technology is surprisingly underdeveloped. To overcome this technological dilemma, establishing a new concept or paradigm for construction of functional materials from nanoscale building units has been long awaited.

Significant efforts have been made to synthesize various nanomaterials including nanoparticles, nanotubes, nanosheets and nanoporous materials. ${ }^{1-3}$ However, their individual functions are limited compared with the huge potential available from integrated systems.
Architecting nanomaterials into hierarchic and organized structures is a rational way to construct advanced functional materials. Recently, we have coined the term 'materials nanoarchitectonics' to express this necessary innovation. (This terminology was first proposed by Dr Masakazu Aono at 1st international symposium on nanoarchitectonics using suprainteractions (NASI-1) at Tsukuba in 2000.) $)^{4-6}$

Forming materials precisely from atoms and molecules into complex three-dimensional systems remains a difficult matter. Of several possible strategies, forming nanomaterials into controlled layer structures could be an easily achievable approach because twodimensional nanomaterials such as nanosheets and layering methodology including the Langmuir-Blodgett (LB) technique ${ }^{7,8}$ and layerby-layer (LbL) assembly 9,10 are currently available. For example, individual nanosheets have sub-nanometric thicknesses and possess different properties depending on their elemental compositions. ${ }^{11}$ Carefully designed assembly of atomically thin nanosheets within LbL structures of precisely controlled sequences and layer spacings leads to novel materials whose properties depart from those of the component nanosheets and/or the corresponding bulk materials. Alternating assembly of two kinds of paraelectric sheets $\left(\mathrm{LaNb}_{2} \mathrm{O}_{7}\right.$ and $\mathrm{Ca}_{2} \mathrm{Nb}_{3} \mathrm{O}_{10}$ ) leads to a ferroelectric material due to effective

\footnotetext{
${ }^{1}$ World Premier International (WPI) Research Center for Materials Nanoarchitectonics (MANA), National Institute for Materials Science (NIMS), Tsukuba, Japan and 2Japan Science and Technology Agency (JST), Tsukuba, Japan

Correspondence: Dr K Ariga, WPI Research Center for Materials Nanoarchitectonics (MANA), National Institute for Materials Science (NIMS), 1-1 Namiki, Tsukuba, Ibaraki 305-0044, Japan.

E-mail: ARIGA.Katsuhiko@nims.go.jp

Received 12 March 2012; revised 1 April 2012; accepted 9 April 2012
} 
formation of heterointerfaces (see later for details). Assembled nanosheets can also be used as an ultrathin window coating with self-cleaning properties. In other examples, assembling nanoporous materials in layered structures has led to the development of highly selective sensors and auto-starting drug delivery systems (see later for details). Fixing nanomaterials into well-designed organized layered structures is an effective way for creation of functional materials.

In this review, we will introduce recent research to develop functional materials by architecting nanomaterials into organized structures, especially in well-controlled layered motifs. Recent rapid progress in layered architectures of other nanomaterials, such as nanoparticles, nanotubes and even bionanomaterials, are comprehensively summarized in this review by selection of very recent examples. Finally, a brief summary of related concepts including atom/molecular-controlled systems and novel design of individual nanomaterials is described to illustrate the potential of nanoarchitectonics in materials science and technology.

\section{HOW TO STRUCTURE FUNCTIONAL LAYERS}

Preparation of functional thin-layer materials is a continuing research topic. ${ }^{12,13}$ In particular, engineering processes aimed at scaleup of ultrathin film preparation has been investigated from the point-ofview of future practical applications. For example, Yan et al. ${ }^{14}$ reported epitaxial formation of bilayer Bernal graphene on copper foil through chemical vapor deposition process. At developing graphene nuclei, edges with dangling bonds are much more reactive to covalently bond with incoming atoms or fragments than the inert face, inducing much faster lateral growth than the vertical counterpart. Incoming carbon radicals are likely to arrange into a thermodynamically stable Bernal stacking structure. Bae et al. ${ }^{15}$ have developed a roll-to-roll process to prepare a predominantly monolayer 30-inch graphene film on a copper substrate by using a chemical vapor deposition process (Figure 1a). The graphene film grown on copper foil was first attached to a thin polymer film through rollers and the copper layer was subsequently removed by electrochemical reaction. The graphene film was finally transferred from the polymer support to a target substrate. This method could be applied for the production of large-scale transparent electrodes.
Chanda et al. ${ }^{16}$ proposed a simple printing approach for large-area, high-quality negative-index metamaterials. As shown in Figure 1b, a silicon wafer with nanoscale patterns of surface relief can be used as a reusable stamp, which is inked with a solid, multilayer stack through electron-beam evaporation. A high-throughput process is completed by transfer printing the ink material onto substrates.

These physical processes are powerful methods for deposition of thermally stable materials but are not always available for fragile and/ or nonvolatile substances. In the latter case, soft processing for controlled layer fabrication becomes crucial and a typical soft layering process might be LB method and LbL assembly. Figure 2a illustrates the concept of the LB process. The LB method was initially proposed for surfactant-like amphiphilic molecules capable of forming a monomolecular layer (monolayer) on a water subphase, which can then be transferred onto a solid substrate in LbL manner. As is widely known, this strategy is mostly used for deposition of organic materials including biopolymers although recent efforts have demonstrated use of the LB method for inorganic substances such as nanorods, nanosheets and other nano-objects. ${ }^{17,18}$ For example, Zeng et al. ${ }^{19}$ applied LB deposition for ultralarge graphene oxide sheets up to $200 \mathrm{~mm}$ in lateral size that could be converted into a transparent thin film conductor by subsequent reduction and chemical doping. The measured sheet resistance was better than that of chemical vapor deposition-prepared graphene film. Makiura et al. ${ }^{20}$ and Motoyama et al. ${ }^{21}$ reported LbL deposition of metal organic frameworks by using the LB process (Figure 3). Spreading a solution of a free base porphyrin derivative on aqueous $\mathrm{CuCl}_{2}$ solution resulted in monolayer-type metal organic framework structures through interfacial complex formation, which could be converted into a LbL nanoarchitecture with intermediate solution rinsing.

These novel examples demonstrate the possibility of extending the LB concept to a wide range of substances. However, LbL assembly has much wider application capabilities because a greater variety of materials than can be applied in the LB method. This approach is one of the most versatile methods for constructing nano-sized layered architectures of various materials including organic polymers, biomaterials, inorganic substances and supramolecular assemblies on both flat substrates (Figure $2 \mathrm{~b})^{22}$ and microscopic colloidal particles
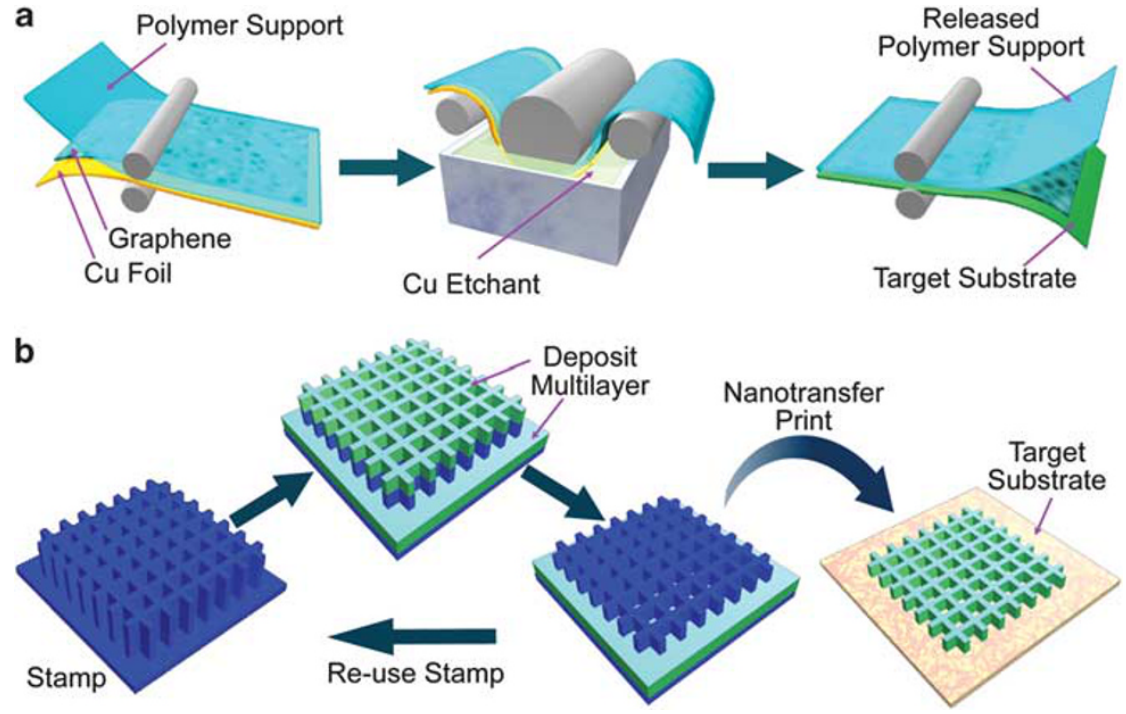

Figure 1 Advanced fabrication methods for functional thin films: (a) roll-to-roll process for large-area graphene film; (b) nanotransfer printing for negative index metamaterials. 
a

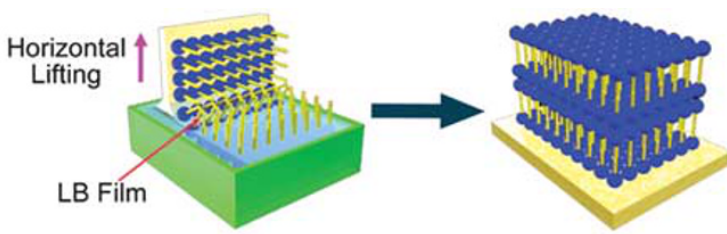

b

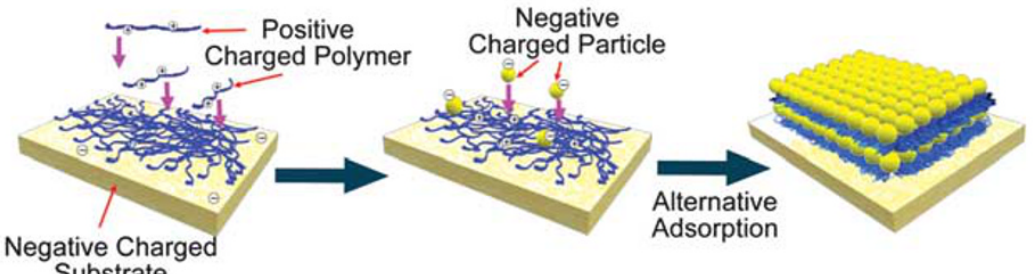

Substrate

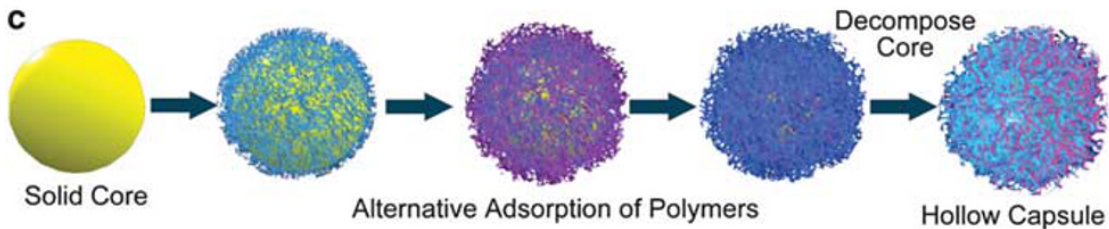

Figure 2 Soft fabrication processes for controlled layered structures: (a) LB method; (b) LbL assembly in flat film mode and (c) LbL assembly in colloidal mode.
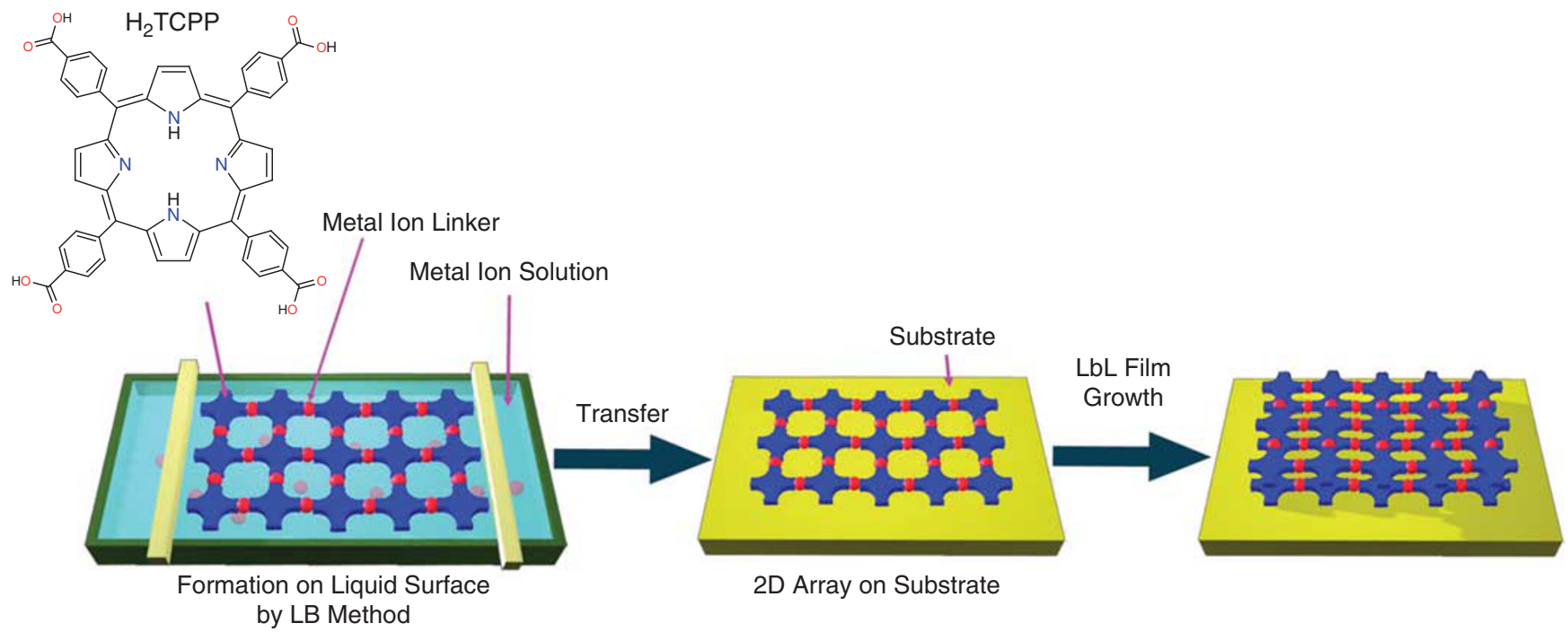

2D Array on Substrate

Figure $3 \mathrm{LbL}$ deposition of metal organic framework using the LB method.

(Figure 2c). ${ }^{23,24}$ Most popular modes of LbL assembly are based on an electrostatic mechanism. As illustrated in Figure 2b, excess adsorption leads to charge neutralization and resaturation in turn leading to charge reversal, resulting in alternation of the surface charge. Continuous assembly between positively and negatively charged materials affords layered architectures with a great freedom in the number of layers and their sequence. Similar processes can be extended to other interactions such as metal coordination, hydrogen bonding, covalent bonding, supramolecular inclusion, bio-specific recognition, charge transfer complex formation and stereo-complex formation. In addition to solution dipping, various techniques including spin coating and spraying have been demonstrated. Because of its great versatility, the LbL technique has become an extremely popular methodology for preparing layered nanoarchitectures.
Therefore, we here focus on recent developments in layered nanoarchitectonics based on LbL assembly.

\section{EXPLORATION OF LBL NANOARCHITECTONICS}

Although LbL has become a major technique for nanostructure preparation, its fundamental aspects are still in the forefront of research. For example, Carrillo and Dobrynin ${ }^{25}$ applied molecular dynamics simulations to multilayer assemblies between oppositely charged nanoparticles on porous materials. Deposition of the nanoparticles has features similar to those observed for conventional LbL assembly on regular planar substrates. For example, the surface overcharge at every deposition step can be estimated as being about half the value of the net charge of the deposited nanoparticles. The amplitude of the charge fluctuations 
decreases as the number of layers increases. The film thickness and surface coverage increase almost linearly with the number of deposition steps. These simulation results almost reproduce what is observed for experimental depositions. On the other hand, Choi et al. $^{26}$ demonstrated the complex nature of LbL assembly by investigating the effect of $\mathrm{pH}$ on LbL film growth of star-shaped polyelectrolytes. Thicknesses and morphologies of the LbL films were governed by either linear or exponential growth mode that can be tuned by varying $\mathrm{pH}$ conditions. Such significant effects of $\mathrm{pH}$ on the structures of LbL assemblies were originally reported by Shiratori and Rubner. ${ }^{27}$ Other parameters, such as charge density, degree of hydration and molecular diffusion, also have significant effects. In addition, a description of polyelectrolyte films violating (almost) all the rules of LbL deposition has been recently published. ${ }^{28}$ Surprisingly, one of the principal authors of this counterintuitive paper is Decher, who is one of the pioneers of LbL technology.

Modification of assembly driving forces has been explored under various demands for expansion of assembly components and

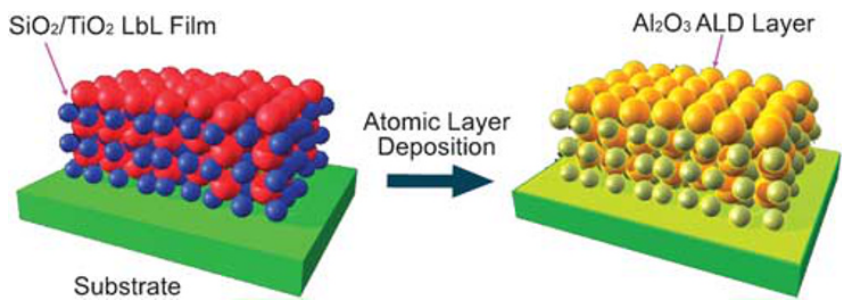

Figure 4 Reinforcement of particle LbL films by atomic layer deposition. improvement of film stability. Dafinone et al. ${ }^{29}$ used atomic layer deposition for mechanical reinforcement of $\mathrm{LbL}$ films of $\mathrm{TiO}_{2}$ and $\mathrm{SiO}_{2}$ nanoparticles. These nanoparticle LbL films had porous structures that were coated with $\mathrm{Al}_{2} \mathrm{O}_{3}$ by atomic layer deposition at low temperature (Figure 4). Robustness of the reinforced nanoparticle LbL films became comparable to that of thermally calcined films. Functionalities specific to the nanoparticle LbL films, such as superhydrophilicity and antireflection properties, were not seriously affected. Reignforcement of the LbL assemblies can be also done through a polymeric network with nanoparticles as demonstrated by Bédard et al. ${ }^{30}$ Shirman et al. ${ }^{31}$ applied halogen bonding for LbL assembly of gold nanoparticles on a planar surface. As illustrated in Figure 5, the surface was first decorated with organic functional groups bearing terminal pyridine moieties that can function as a halogen bond acceptor. Gold nanoparticles functionalized with halogen bond donor ligands were then deposited. By using appropriate linkers with halogen bond acceptors, gold nanparticles could be assembled in a LbL manner. Ligand shape and the number of layers could be used to control the morphology of the films.

To prepare robust LbL films of organic components, assemblies with covalent bond formation have been investigated including methods using 'click' reactions. ${ }^{32}$ However, most of the proposed methods require use of additional chemicals in reactions for covalent bond formation. In contrast, $\mathrm{Li}$ et al. $^{33}$ has invented a novel methodology, electrochemical coupling LbL (ECC-LbL) assembly, that can be used to covalently immobilize organic functional units such as porphyrin, fullerene and fluorene into thin films having desired thicknesses and designable sequences for both homo- and heteroassemblies through ECC of carbazole groups (Figure 6a).

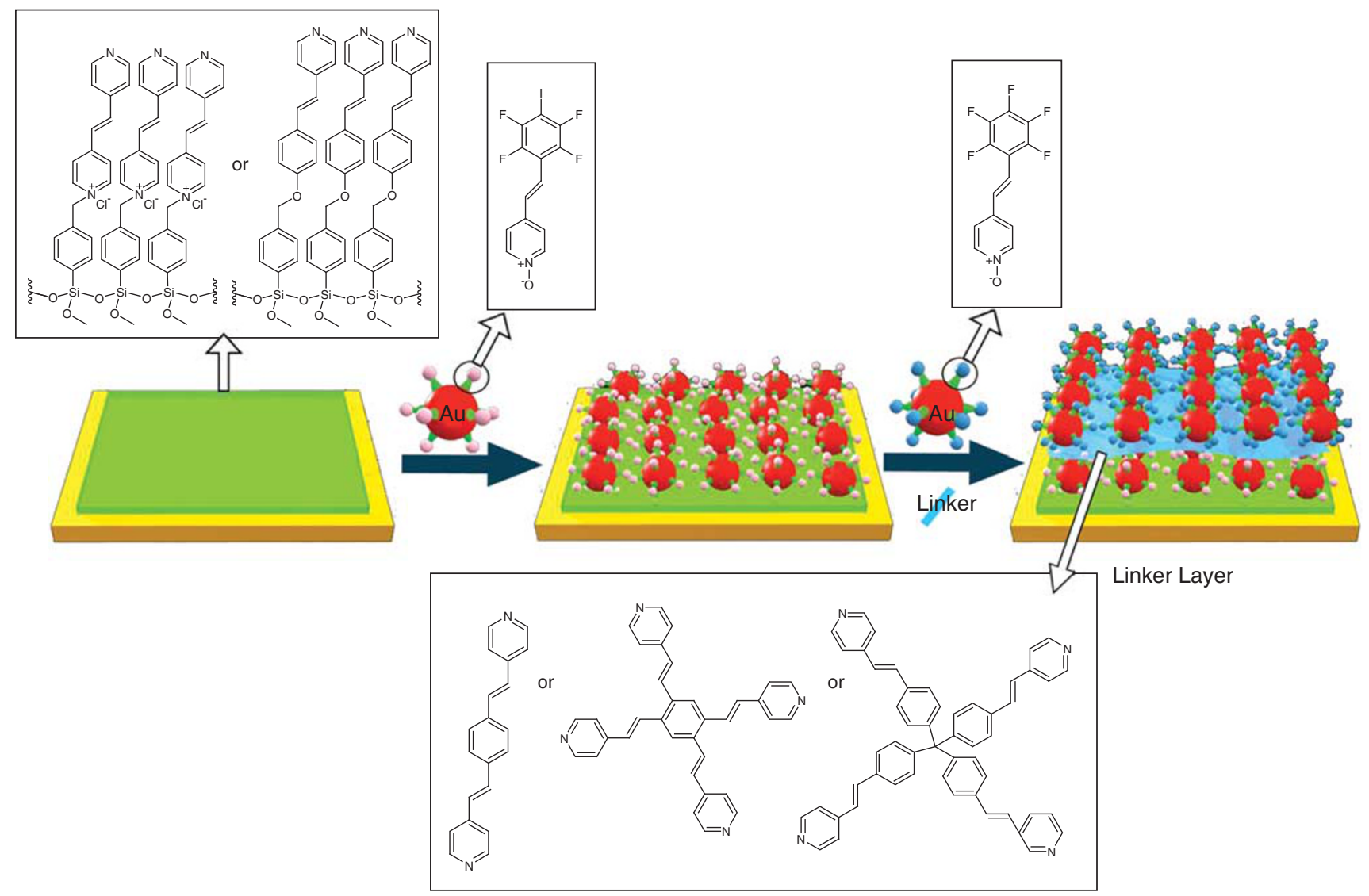

Figure 5 Halogen-bonding-mediated LbL assembly. 


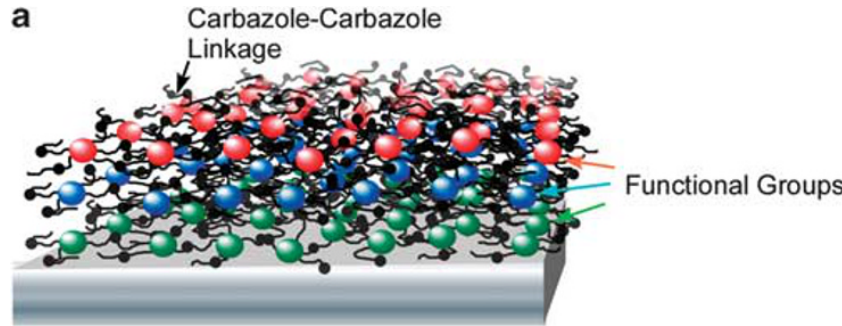

b

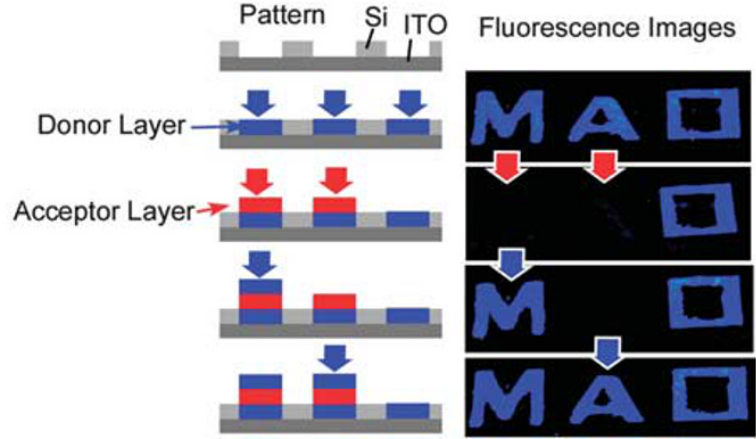

Figure 6 ECC-LbL assembly: (a) fundamental concept; (b) application to controlled display.

As $\mathrm{N}$-alkylcarbazoles and their dimers have large hole transport mobilities, electrochemical signals can be transmitted to the top layers of their films. This clean reagentless process is especially useful for constructing covalently linked layer-controlled thin films on a sensitive device surface. ECC-LbL assembly can also be used in area-selective patterning mode. ${ }^{34}$ As illustrated in Figure $6 \mathrm{~b}$, three capital letters, $\mathrm{M}, \mathrm{A}$ and $\mathrm{O}$, were positioned on different parts of indium tin oxide on the same glass surface where ECC-LbL assembly could be undertaken at different areas and each of the indium tin oxide parts could then be individually toggled on by connection to a working electrode. The patterned display was demonstrated under irradiation with ultraviolet light $(254 \mathrm{~nm})$ in the dark. By modifying the electron donor and electron acceptor components in the layered structures, patterning of a fluorescent display was possible. The ECCLbL approach should be a powerful method for constructing robust and well-designed organic layered structures with potential uses in various types of organic devices.

Recently, Yoon et al. ${ }^{35}$ proposed use of the nucleophilic substitution reaction between bromo and amine groups for LbL assembly on silica colloids with 2-bromo-2-methylpropionic acid-stabilized quantum dots and 2-bromo-2-methylpropionic acid-stabilized iron oxide particles (2-bromo-2-methylpropionic acid- $\mathrm{Fe}_{3} \mathrm{O}_{4}$ ), along with amine-functionalized poly(amidoamine) in organic media. The resulting LbL-coated particles exhibited superparamagnetism, photoluminescence and magneto-optical tuning properties with long-term stability in nonpolar solvents.

\section{PHYSICOCHEMICAL APPLICATIONS}

Fabrication of layered structures is useful for control of interlayer physical interactions and immobilization of functional nanostructures on device surfaces. A typical example of these advantages was demonstrated by Osada et al. ${ }^{36}$ They prepared layered superlattice structures of oxide nanosheets by using LbL assembly. Ferromagnetic nanosheets $\left(\mathrm{Ti}_{0.8} \mathrm{Co}_{0.2} \mathrm{O}_{2}\right.$ and $\left.\mathrm{Ti}_{0.6} \mathrm{Fe}_{0.4} \mathrm{O}_{2}\right)$ prepared by delaminating layered titanates were assembled sequentially on substrates such as atomically flat $\mathrm{SrTiO}_{3}\left(\mathrm{Nb} 0.5 \mathrm{wt} \% ; \mathrm{SrTiO}_{3}: \mathrm{Nb}\right)$ or quartz glass chips,

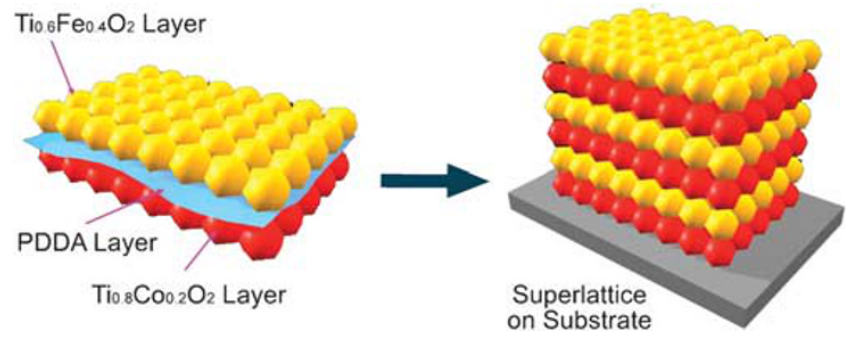

Figure 7 Superlattice of oxide nanosheets LbL film.
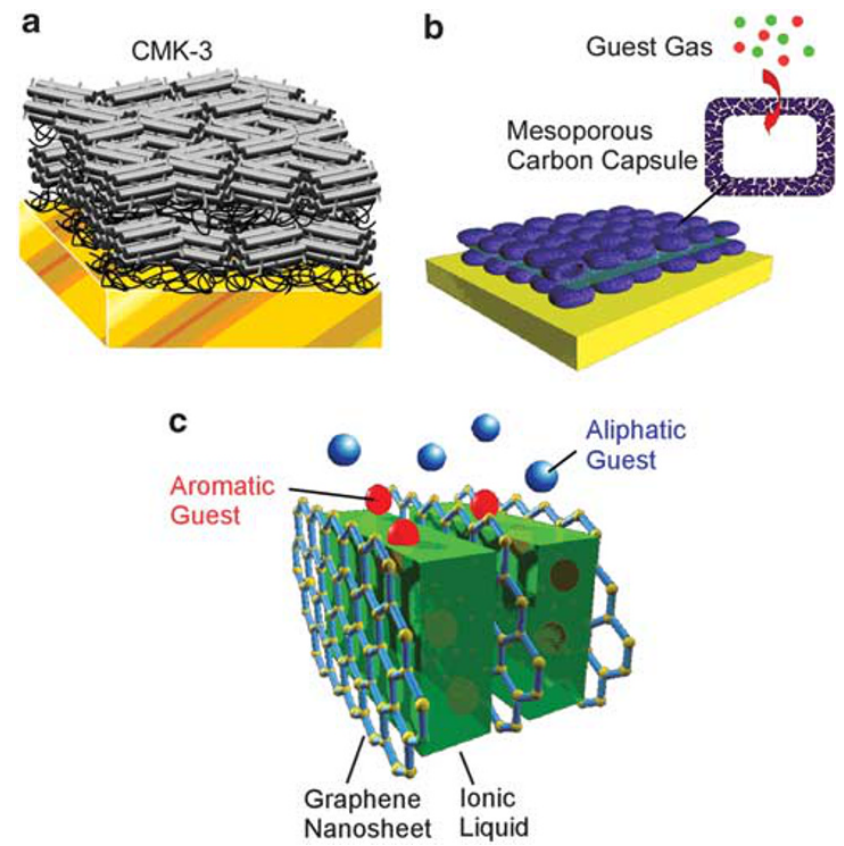

Figure 8 Sensors based on LbL films of inorganic nanostructures: (a) an LbL film of mesoporous carbon CMK-3; (b) an LbL film of mesoporous carbon capsule and (c) an LbL film of graphene nanosheet and ionic liquid.

together with the polymer cation, poly(diallyldimethylammonium chloride; (Figure 7). The superlattice structures exhibited a gigantic magneto-optical response in the visible wavelength region. This response originates from the interlayer $d-d$ transitions $\left(\mathrm{Co}^{2+}\right.$ $\mathrm{Fe}^{3+}$ ) between adjacent nanosheets. In this superlattice, which can be regarded as an artificial ferromagnet, the charge, spin and orbital degrees of freedom might be strongly coupled at the interface. Layering combinations can be created with infinite varieties and artificial layered structures with enhanced interface interactions can be formed with a great freedom of design.

Immobilization of nanostructured materials as thin films on sensor device surfaces would be a potentially useful means of revealing the properties specific to nanostructures for sensing responses. The following examples demonstrate advantages of the LbL assembly for sensing applications. Ariga et al. ${ }^{37}$ assembled mesoporous carbon (CMK-3) with regular pores on a mass-sensitive device, a quartz crystal microbalance (QCM), by LbL assembly with the aid of polyelectrolytes (Figure $8 \mathrm{a}$ ). The resulting sensors were used to discriminate tea components in an aqueous phase. Sensor responses (frequency changes) for tannic acid easily exceeded those observed for catechin and caffeine. The superior adsorption capacity for tannic acid likely originates in its molecular structure, that is, multiple phenyl rings of the tannic acid molecule can interact with the carbon 
surface through $\pi-\pi$ interactions and hydrophobic effects as well as size fitting of tannic acid to the CMK-3 nanochannel. The observed behavior may be similarly explained by enhanced guest-guest interactions under confined geometry. These observations should improve our understanding of molecular interactions within nanospaces.

As demonstrated in the previous example, assembling rather unusual nanostructures into thin films can be performed using LbL assembly. The next example shown in Figure $8 \mathrm{~b}$ illustrates a gas sensor with tunable selectivity prepared by LbL assembly of mesoporous carbon capsules on a QCM plate. ${ }^{38}$ The carbon capsules of homogeneous dimensions $\left(1000 \times 700 \times 300 \mathrm{~nm}^{3}\right)$ with $35-\mathrm{nm}$ thick mesoporous walls $(4.3 \mathrm{~nm}$ in diameter) were synthesized using zeolite crystals as templates, and were assembled in LbL manner after coating the capsules with surfactants. Various volatile substances could be discriminated by observing the in situ frequency decrease of the QCM sensor where aromatic hydrocarbons such as benzene and toluene were generally more easily detected than aliphatic hydrocarbons such as cyclohexane. In addition, selectivity of gas adsorption could be flexibly controlled by impregnation of the carbon capsule films with secondary recognition sites. The designable guest selectivity might be utilized for widespread applications including as sensors or filters. Graphene and graphene oxide are attractive materials for the LbL assembly. ${ }^{39}$ As illustrated in Figure $8 \mathrm{c}$, pieces of graphene can be disassembled from graphite then reassembled into LbL structures together with ionic liquid. ${ }^{40}$ Graphene oxide sheets, prepared by oxidization of graphite under acidic conditions, were reduced to graphene sheet in the presence of ionic liquids in water. Composites of grapheme sheet/ionic liquid as charge-decorated nanosheets were assembled alternately with poly(sodium styrenesulfonate) by LbL adsorption on a QCM sensor.
The resulting films showed significantly higher selectivity $(>10$ times) for benzene vapor over cyclohexane despite their similar molecular sizes, molecular weights and vapor pressures. Adsorption of $\mathrm{CO}_{2}$ vapors from a saturated sodium hydrocarbonate solution into the LbL films also showed enhanced response.

There is huge scope for applications of LbL assemblies. Li et al. ${ }^{41}$ used LbL architectonics to prepare coatings capable of extinguishing flames. There are four major requirements for flame retardants: a source of carbon, a source of acid, a blowing agent and a binder to keep the components suspended in a liquid dispersion and form a solid film on a surface. Li et al. assembled poly(sodium phosphate) and poly(acrylic acid) as the blowing agent. The latter poly(acrylic acid degrades into low-molecular-weight compounds, which are released as blowing gas where $\mathrm{NH}_{3}$ reacts with $\mathrm{O}_{2}$ to form $\mathrm{N}_{2}$ and $\mathrm{H}_{2} \mathrm{O}$. The resulting LbL polymer coating can act as an environmentally friendly alternative for protecting fabrics. Zhu et al. developed transparent conductors by LbL assembly of carbon nanotubes. ${ }^{42}$ Conventional transparent conductors based on coating of carbon nanotubes and related materials have several deficiencies such as poor mechanical properties, significant roughness, lowtemperature resilience and rapid loss of conductivity. To overcome these difficulties, LbL assembly of hydroethyl cellulose and sulfonated polyetheretherketone-coated carbon nanotubes was performed (Figure 9). Hydroethyl cellulose and sulfonated polyetheretherketone function as molecular glue and nanotube stabilizer, respectively, and they also serve as dopants. Doping is based on electron transfer from several valence bands of carbon nanotubes to low lying unoccupied levels of sulfonated polyetheretherketone in the $\pi$-stacked electronic system. A cumulative electrical/optical/mechanical performance expressed as figure of merit exceeds that of indium tin oxide.

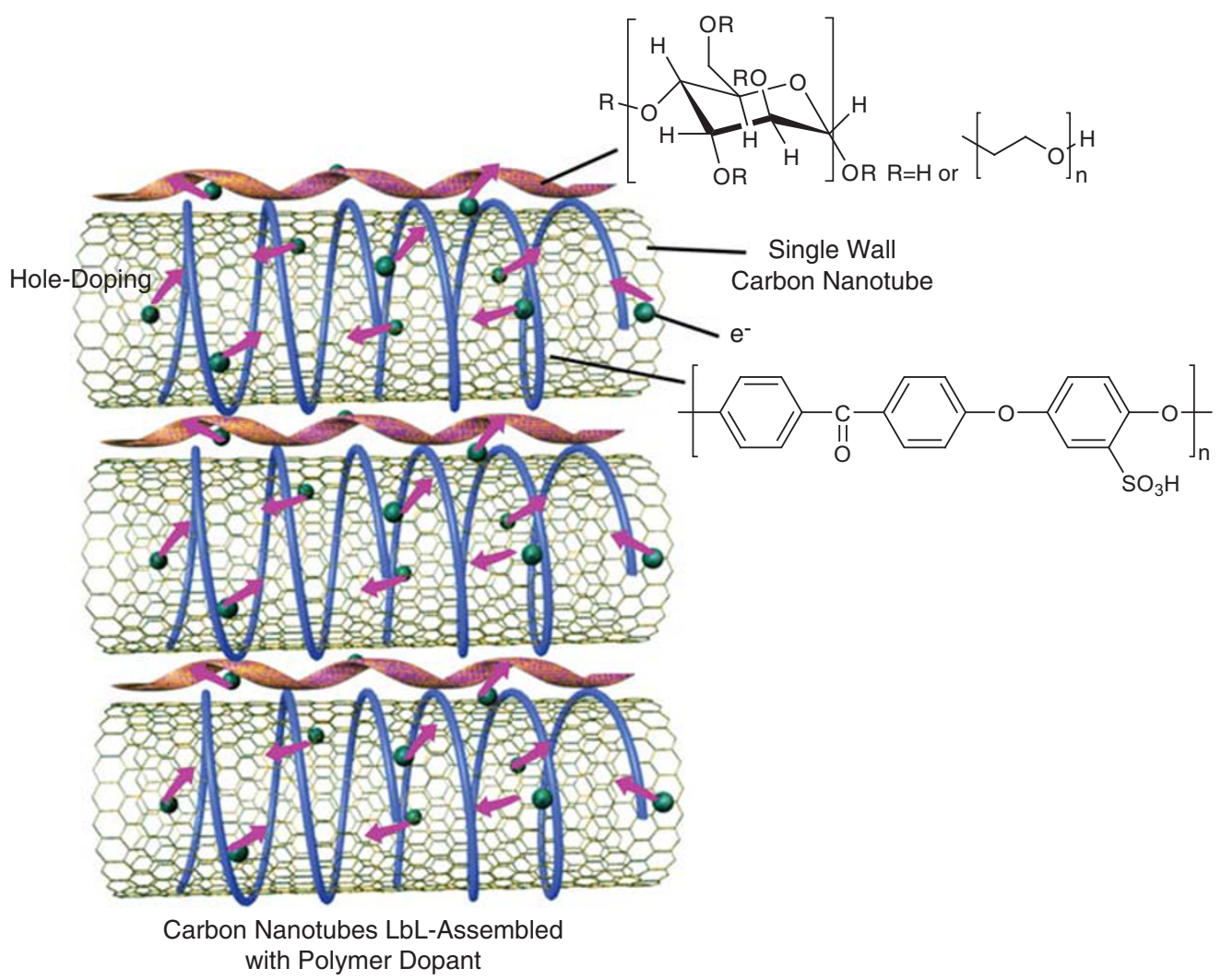

Figure 9 Wrapping of carbon nanotube for $L b L$ assembly. 
Li et al. ${ }^{43}$ reported a strategy for preparing materials for electronics with the LbL technique. They first assembled graphene oxide and polyoxometalate clusters of $\mathrm{H}_{3} \mathrm{PW}_{12} \mathrm{O}_{40}$ into multilayer films by LbL assembly. Ultraviolet irradiation induced photoreduction of the films, which reduce graphene oxide due to the photocatalytic activity of the polyoxometalate clusters. This strategy is useful for fabrication of reduced graphene oxide films with precisely controlled thicknesses on various substrates. They demonstrated preparation of field effect transistors based on the composite films where typical ambipolar features and good transport properties for both holes and electrons were observed. They expect that a rational combination of the luminescent property of polyoxometalate clusters with the electric response of reduced graphene oxide films could lead to logic-gate devices with dual optical and electrical output functions. Ha et al. ${ }^{44}$ demonstrated preparation of self-assembled nanodielectrics with alternate structures of organic and inorganic layers, where polarizable, phosphonic acid-functionalized organic precursors and ultrathin layers of high-k inorganic oxide materials were used (Figure 10). The ultrathin dielectric materials obtained were adherent, insulating, high-capacitance and thermally stable. They could be applied for low-operating voltage organic and inorganic semiconductor-based thin film transistor devices as versatile gate dielectrics with excellent thermal stability.

Lee et $a l .{ }^{45}$ used the LbL technique for fabrication of electrodes with additive-free, densely packed and functionalized multiwalled carbon nanotubes. The resulting electrodes exhibited high gravimetric energy delivered at an exceptionally high power of lithium battery cells. In particular, thicker large-scale electrodes of $10 \mu \mathrm{m}$ size could be produced using a spray LbL method to reduce assembly time markedly. Figure 11 exemplifies a unique application of LbL objects where energetic walking devices based on polyelectrolyte multilayered films are demonstrated. ${ }^{46}$ This innovative work was performed by Sun and coworkers who made actuators by attaching LbL bilayers of poly(acrylic acid/poly(allylamine hydrochloride) onto ultravioletcured Norland Optical Adhesive 63 substrate (Norland Products Inc., Cranbury, NJ, USA). This moisture-sensitive actuator was located between two pieces of polyethylene terephthalate. The actuator drove unidirectional walking of the device on a ratchet substrate upon control of relative humidity. When relative humidity was set at high
(40\%), the walking device bent upward into an arch due to swelling of the PAA/poly(allylamine hydrochloride LbL layer. This moisturesensitive part was in turn contracted by decreasing relative humidity to $11 \%$. The latter change caused stretching of the walking device forward in the designated direction because the fore claw moved forward and the hind claw was fixed by one of the indentations on the substrate. This kind of actuator might be used to build miniaturized devices capable of rapid response to a wide variety of stimuli.

\section{BIOMEDICAL APPLICATIONS}

As LbL assembly can be conducted under mild conditions compared with other thin film preparation techniques, use of bio-related materials and application in biomedical fields can illustrate the unique advantages of the LbL assembly. ${ }^{47-49}$ In this section, recent examples of bio-related and biomedical applications of LbL are described.

Komatsu et al. ${ }^{50}$ designed nanotubes of human serum albumin as a virus trap (Figure 12). The nanotubes were prepared by LbL assembly of human serum albumin and poly(L-arginine) into a nanoporous polycarbonate membrane, followed by depositions of poly(L-glutamic acid) and anti-hepatitis B surface antibody-antibody. Removal of porous polycarbonate template by selective dissolution resulted in
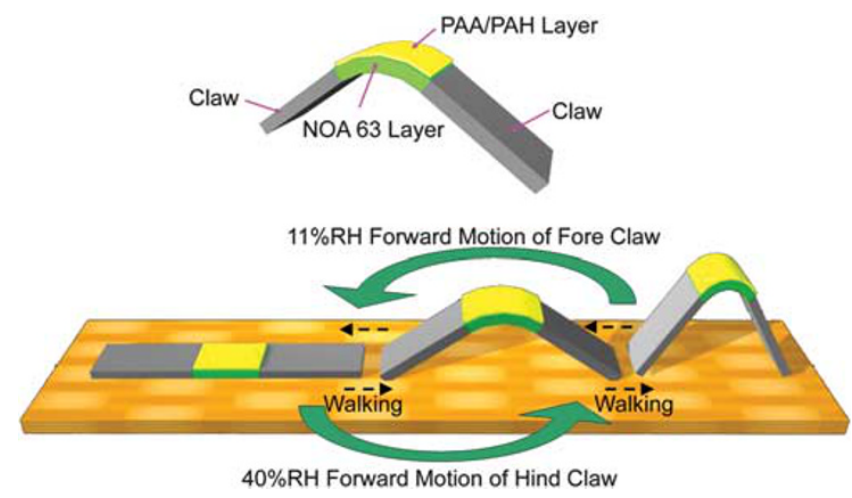

Figure 11 Energetic walking device based on LbL structures.

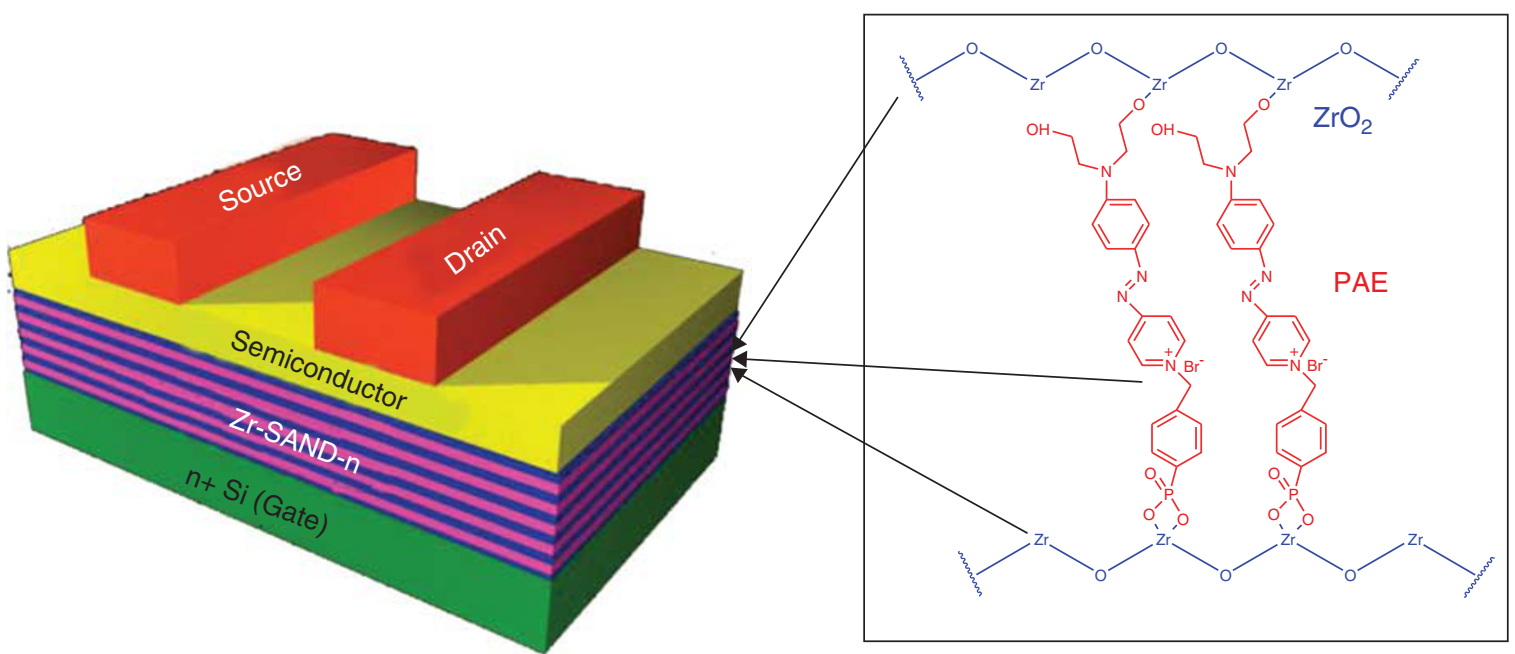

Bottom-Gate Top-Contact Thin-Film Transistor (TFT) Device

Figure 10 Organic inorganic LbL assembly as a thin film transistor. 


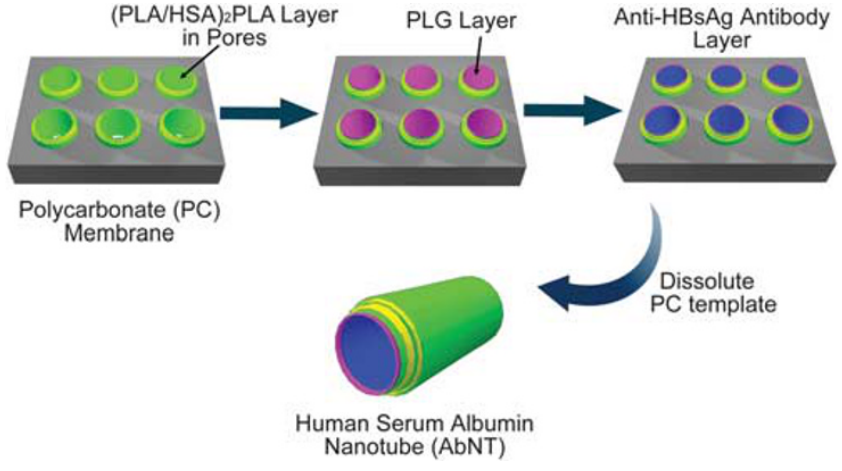

Figure $12 \mathrm{LbL}$ nanotubes of human serum albmin as a virus trap.

multilayered protein nanotubes having an anti-hepatitis B surface antigen antibody (hepatitis B surface antibody) layer as an internal wall. The amount of genome DNA in the hepatitis B virus solution markedly decreased after incubation with the synthesized nanotubes. This result suggested that the infectious particles were completely entrapped in the one-dimensional pore space of the LbL nanotubes. The efficiency of removal by a single nanotube treatment reached 3.9 $\log$ order. The authors anticipated that this kind of would be used as a novel type of virus detection and removal apparatus.

Exploration of drug delivery systems is undoubtedly an important subject in biomedical applications. The LbL method has been used to make significant contributions to this field as biomaterials and biocompatible materials can be easily immobilized and nanostructured materials appropriate for drug carriers can be embedded into thin films. Ji et al. ${ }^{51,52}$ developed LbL films with unique materials' release capabilities. As illustrated in Figure 13, the films used included regular-sized mesoporous capsules as materials' carriers. Anionic mesoporous silica capsules and cationic polyelectrolyte were deposited on a QCM sensor using LbL assembly with the aid of anionic silica nanoparticles as a co-adsorber. Quantitative analyses on evaporation of entrapped water within capsules in the LbL films under a variety of conditions were investigated. An increase in QCM frequency corresponds to a decrease in mass and, in this case, is symptomatic of water release from the interior of the silica capsule within the LbL film. The frequency shifts upon water evaporation from the mesoporous LbL films possess a stepwise profile even though no external stimulus was applied. The observed stepwise release of water is assumed to originate from combination of two processes: water evaporation from the pores and capillary penetration into the pores. Initially, water entrapped in mesopore channels evaporates to the exterior, which is observed as the first step of water release. After most of the water has evaporated from the mesopore channels, water enters that region from the capsule interior probably through rapid capillary penetration. Water evaporation rate at each step can be controlled by several factors such as temperature and the co-adduct materials (silica particle and polymer). This release profile was also used in a demonstration of controlled release of various fluid drugs such as fragrance molecules. It is a rare example of a stimulusfree controlled release medium, which operates in a stepwise manner with prolonged release efficiency, a feature useful for controlledrelease drug delivery. Similarly, importance of hierarchic structures for drug delivery was mentioned with the novel concept of multicompartmental micro- and nanocapsules by Delcea et al. ${ }^{53}$

Instead of drug delivery from substrates, LbL objects themselves have been widely used as drug carriers. Cui et al..$^{54}$ reported preparation of LbL microcapsules that can carry hydrophobic drugs.

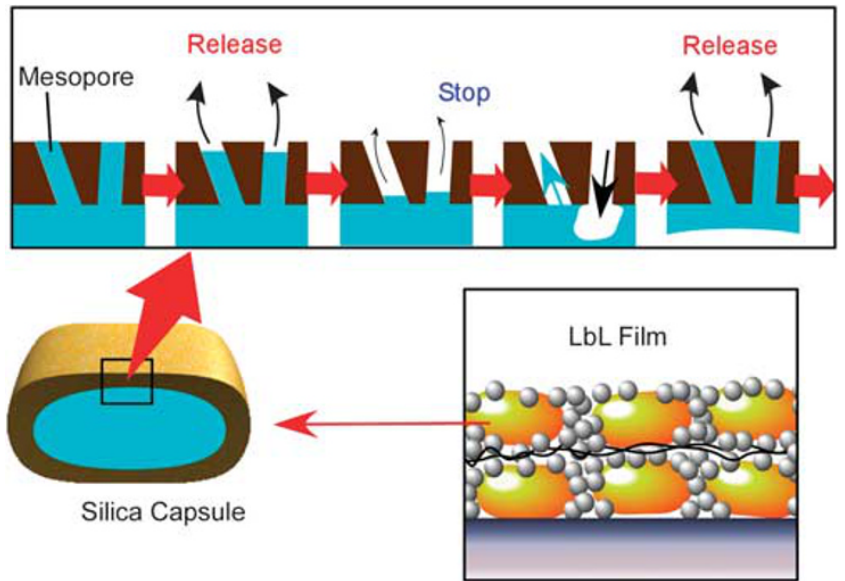

Figure 13 Auto-modulated drug delivery system made from LbL assembly of mesoporous silica capsules.

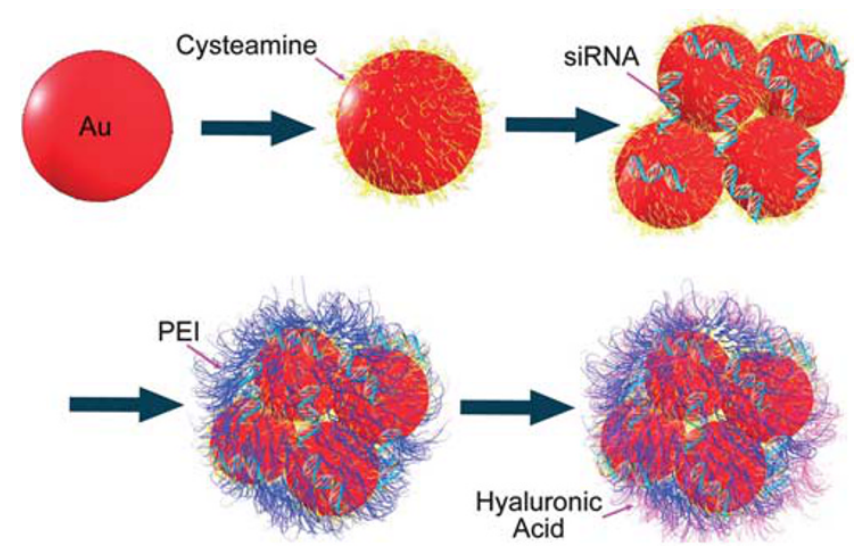

Figure $14 \mathrm{LbL}$ nanocomplex of gold nanoparticles, cystamine, and si-RNA, and hyaluronic acid.

They used alkylated hyaluronic acid as a hydrophobic components that can be assembled with poly(L-lysine) into hollow capsules. The resulting capsules can trap hydrophobic drugs and the entrapped drugs have a very high stability in a physiological medium. Lee et al. ${ }^{55}$ developed LbL nanocomplex including gold nanoparticles, cystamine, small interfering RNA and hyaluronic acid. As illustrated in Figure 14, cysteamine-modified gold nanoparticles were sequentially modified with small interfering RNA, poly(ethyleneimine) and hyaluronic acid. The nanocomplex obtained was used for target-specific drug delivery to B16F1 cells with receptors for hyaluronic acid. When apolipoprotein B small interfering RNA as a model drug was used, apolipoprotein B mRNA level in the liver tissue was markedly reduced. Poon et al. ${ }^{56}$ developed LbL nanoparticles with appropriately designed layered structures for targeting of tumor hypoxia. The capsules were coated with a stealth layer of poly(ethylene glycol) to suppress nonspecific cellular uptake of positively charged LbL nanoparticles. This poly(ethylene glycol) layer can be selectively removed by acidity generated in the hypoxic tumor microenvironment. Capsules with exposed cationic charges were readily taken up by tumor cells. Control of shielding and deshielding of charges on LbL nanoparticles enabled targeting of drug deliver to tumor cells.

An LbL coating is also useful for cell engineering including cell modifications and cell assembly. Wilson et al. ${ }^{57}$ assembled LbL 
films of poly(L-lysine)-graft-poly(ethylene glycol) and alginate as polyelectrolyte multilayer on living cells. The coated cells maintained viability and function upon transplantation and were used as carriers for in vivo delivery of multilayer-containing biomolecular payloads. Yang et al..$^{58}$ demonstrated a method for covering cells with silica shell with the aid of LbL films. As illustrated in Figure 15, a yeast cell was first coated with polyelectrolyte LbL films and then a silica shell was fabricated by polycondensation of thiolfunctionalized alkoxysilane. The thiol groups introduced at the surface operate as starting points for introduction of various functional moieties such as fluorescein and streptavidin. The authors expected that the formation of artificial spores on cells would be useful as cellbased sensors, reactors, devices and single-cell-based biology. Nishiguchi et al..$^{59}$ developed the cell-accumulation technique (Figure 16). A single cell was first coated with LbL films of fibronectin and gelatin. The fibronectin-gelatin films can interact with integrin receptors of the cell membrane resulting in the cell-cell adhesion of all seeded cells in three-dimensions at the same time. The formation of a large diversity of layered cell societies is possible just by controlling the layer number, cell type and location. This simple cell archtecture approach is expected to be promising methodology for the in vitro construction of tissue or organ models.

\section{FUTURE DIRECTION: EXTENSION TO VARIOUS NANOARCHITECHTONICS}

In this review, we have briefly overviewed recent research on layered nanoarchitectonics for innovative materials based on the LbL assembly technique. Some of the examples indicate the potential importance of architectonics at the nanoscale where materials' compositions and functions can be markedly altered from the original bulk materials. For example, single-layered nanosheets can be disassembled from the bulk layered materials and re-architected into layered structures in predesignated combinations and layering sequences. Artificially structured layered objects are capable of expressing totally novel properties based on creation of new interfaces at the nanoscale. Graphite can be disassembled into graphene

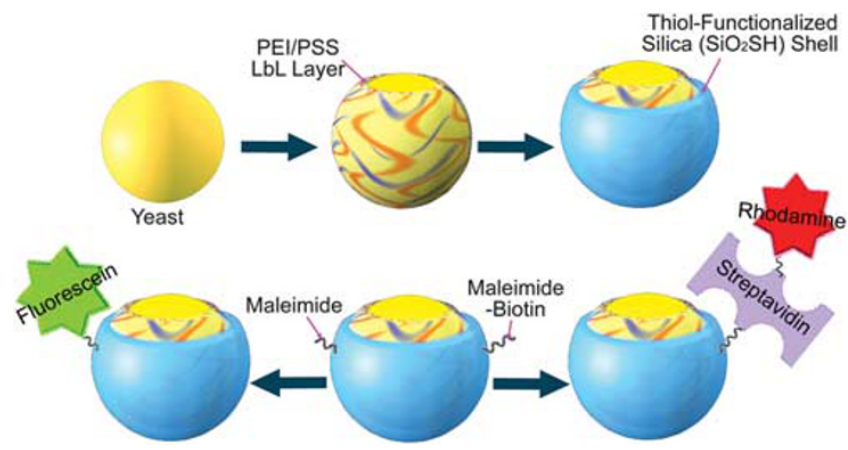

Figure $15 \mathrm{LbL}$ coating of yeast cell with surface functionalization.

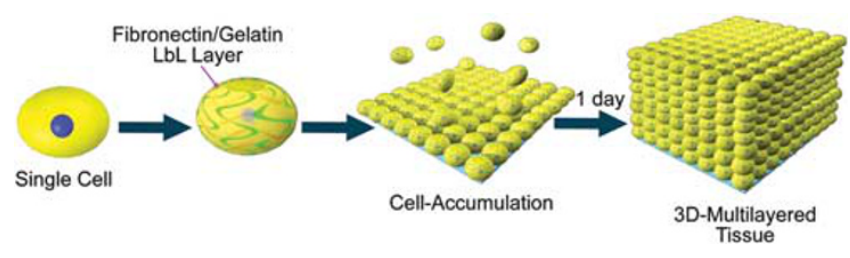

Figure 16 Cell accumulation through LbL coating. sheets and/or graphene oxide sheets. The disassembled pieces can be reconstituted with other components such as ionic liquids to produce novel layered nanostructures with sensing capabilities. These efforts clearly demonstrate that properties of the original materials can be altered by nanoarchitectonics. In addition, recent research on the LbL assembly ${ }^{60-62}$ revealed further advances in nanoarchtectonics including specific patterning, ${ }^{63}$ biomaterial template synthesis ${ }^{64,65}$ and porous membrane fabrication. ${ }^{66}$

The concept of layered nanoarchitectonics can be applied over a variety of length scales, that is, from atomic scale architecture, such as atom-layer deposition and graphene-sheet fabrication, to large-scale assembly including cell society accumulation. In fact, methodologies can be expanded to include microscopic and visible scales from the nanoworld. The nanoarchitectonics concept can be applied in all dimensions. For example, control of deposition of atoms and bridging molecules in three-dimensionally architected structures can create novel device concepts such as atomic switches ${ }^{67-69}$ and single molecule logic gate. ${ }^{70,71}$ Architecting atoms by rational means into materials has resulted in novel and highly enhanced functions as can be seen in photocatalysts ${ }^{72,73}$ and spintronics materials. ${ }^{74,75}$ Forming stimuli-responsive materials with softly deformable structures can lead to the development of innovative and unusual functional materials such as self-powered sensors ${ }^{76}$ and hand-operatable molecular screening systems. ${ }^{77,78}$ In these examples, functional units and elements are structured within rational designs to create new functions that cannot be obtained by simple combinations of the properties of the original materials in their bulk states.

Most of these strategies have been conducted as parts of established research fields such as nanotechnology or materials science. However, it is not a good idea to categorize these new technologies into old families, as their importance may be neglected or misunderstood. To apply the advanced knowledge available from nanotechnology to materials science, we need to make a conceptual breakthrough. Nanotechnology has revealed the nanoscale world. Next, nanoarchitectonics should be used to build up innovative materials and systems based on the products of nanotechnology. Although there are lots of techniques for constructing small objects, including selfassembly and sophisticated fabrications, these separately developed methodologies have to be unified to one concept for the next step, this being nanoarchitectonics. Thus, it may be easy to appreciate that the conceptual evolution from nanotechnology to nanoarchitectonics is very similar to the process of disassembling bulk materials to nanostructures and re-architecting nanostructures into novel materials, that is, nanotechnology disassembles materials and nanoacchitectonics will be used to re-architect them into more useful forms.

1 Ma, R. \& Sasaki, T. Nanosheets of oxides and hydroxides: ultimate 2D charge-bearing functional crystallites. Adv. Mater. 22, 5082-5104 (2010).

2 Ariga, K., Vinu, A., Yamauchi, Y., Ji, Q. \& Hill, J. P. Nanoarchitectonics for mesoporous materials. Bull. Chem. Soc. Jpn. 85, 1-32 (2012).

3 Golberg, D., Costa, P. M. F. J., Wang, M.-S., Wei, X., Tang, D.-M., Xu, Z., Huang, Y. Gautam, U. K., Liu, B., Zeng, H., Kawamoto, N., Zhi, C., Mitome, M. \& Bando, Y. Nanomaterial engineering and property studies in a transmission electron microscope. Adv. Mater. 24, 177-194 (2012).

4 Weiss, P. S. A Conversation with Dr Masakazu Aono: I eader in atomic-scale control and nanomanipulation. ACS Nano 1, 379-383 (2007).

5 Ariga, K., Li, M., Richards, G. J. \& Hill, J. P. Nanoarchitectonics: a conceptual paradigm for design and synthesis of dimension-controlled functional nanomaterials. J. Nanosci. Nanotechnol. 11, 1-13 (2011).

6 Aono, M., Bando, Y. \& Ariga, K. Nanoarchitectonics: pioneering a new paradigm for nanotechnology in materials development. Adv. Mater. 24, 150-151 (2012).

7 Michinobu, T., Shinoda, S., Nakanishi, T., Hill, J. P., Fujii, K., Player, T. N., Tsukube, H. \& Ariga, K. Langmuir monolayer of cholesterol-armed cyclen complex that can 
control enantioselectivity of amino acid recognition by surface pressure. Phys. Chem Chem. Phys. 13, 4895-4900 (2011).

8 Ariga, K., Mori, T. \& Hill, J. P. Evolution of molecular machines: from solution to soft matter interface. Soft Matter 8, 15-20 (2012).

9 Decher, G. Fuzzy nanoassemblies: toward layered polymeric multicomposites. Science 277, 1232-1237 (1997).

10 Ariga, K., Hill, J. P. \& Ji, Q. Layer-by-layer assembly as a versatile bottom-up nanofabrication technique for exploratory research and realistic application. Phys. Chem. Chem. Phys. 9, 2319-2340 (2007).

11 Osada, M. \& Sasaki, T. Two-dimensional dielectric nanosheets: novel nanoelectronics from nanocrystal building blocks. Adv. Mater 24, 210-228 (2012)

12 Ariga, K., Lee, M. V., Mori, T., Yu, X.-Y. \& Hill, J. P. Two-dimensional nanoarchitectonics based on self-assembly. Adv. Colloid Interface Sci. 154, 20-29 (2010).

13 Sakakibara, K., Hill, J. P. \& Ariga, K. Thin-film-based nanoarchitectures for soft matter controlled assemblies into two-dimensional worlds. Small 7, 1288-1308 (2011).

14 Yan, K., Peng, H., Zhou, Y., Li, H. \& Liu, Z. Formation of bilayer Bernal graphene: layerby-layer epitaxy via chemical vapor deposition. Nano Lett. 11, 1106-1110 (2011).

15 Bae, S., Kim, H., Lee, Y, Xu, X, Park, J.-S., Zheng, Y., Balakrishnan, J., Lei, T., Kim, H. R., Song, Y. I., Kim, Y.-J., Kim, K. S., Özyilmaz, B., Ahn, J.-H., Hong, B. H. \& lijima, S Roll-to-roll production of 30 -inch graphene films for transparent electrodes. Nat. Nanotechnol. 5, 574-578 (2010).

16 Chanda, D., Shigeta, K., Gupta, S., Cain, T., Carlson, A., Mihi, A., Baca, A. J., Bogart G. R., Braun, P. \& Rogers, J. A. Large-area flexible 3D optical negative index metamaterial formed by nanotransfer printing. Nat. Nanotechnol. 6, 402-407 (2011).

17 Acharya, S., Hill, J. P. \& Ariga, K. Soft Langmuir-Blodgett technique for hard nanomaterials. Adv. Mater. 21, 2959-2981 (2009).

18 Acharaya, S., Shundo, A., Hill, J. P. \& Ariga, K. Langmuir films of unusual components. J. Nanosci. Nanotechnol. 9, 3-18 (2009).

19 Zheng, Q., Ip, W. H., Lin, X., Yousefi, N., Yeung, K. K., Li, Z. \& Kim, J.-K. Transparent conductive films consisting of ultralarge graphene sheets produced by LangmuirBlodgett assembly. ACS Nano 5, 6039-6051 (2011).

20 Makiura, R., Motoyama, S., Umemura, Y., Yamanaka, H., Sakata, O. \& Kitagawa, H. Surface nano-architecture of a metal-organic framework. Nat. Mater. 9, 565-571 (2010).

21 Motoyama, S., Makiura, R., Sakata, O. \& Kitagawa, H. Highly crystalline nanofilm by layering of porphyrin metal-organic framework sheets. J. Am. Chem. Soc. 133, 56405643 (2011)

22 Hammond, P. T. Form and function in multilayer assembly: new applications at the nanoscale. Adv. Mater. 16, 1271-1293 (2004).

23 Caruso, F. Nanoengineering of particle surfaces. Adv. Mater. 13, 11-22 (2001).

24 Delcea, M., Möhwald, H. \& Skirtach, A. G. Stimuli-responsive LbL capsules and nanoshells for drug delivery. Adv. Drug. Deliv. Rev. 63, 730-747 (2011).

25 Carrillo, J.-M. Y., \& Dobrynin, A. V. Layer-by-layer assembly of charged nanoparticles on porous substrates: molecular dynamics simulations. ACS Nano 5, 3010-3019 (2011).

26 Choi, I., Suntivich, R., Plamper, F. A., Synatschke, C. V., Müller, A. H. E. \& Tsukruk, V. $\mathrm{V}$. $\mathrm{pH}$-Controlled exponential and linear growing modes of layer-by-layer assemblies of star polyelectrolytes. J. Am. Chem. Soc. 133, 9592-9606 (2011).

27 Shiratori, S. S. \& Rubner, M. F. pH-Dependent thickness behavior of sequentially adsorbed layers of weak polyelectrolytes. Macromolecules 33, 4213-4219 (2000).

28 Cini, N., Tulun, T., Decher, G. \& Ball, V. Step-by-step assembly of self-patterning polyelectrolyte films violating (almost) all rules of layer-by-layer deposition. J. Am. Chem. Soc 132, 8264-8265 (2010).

29 Dafinone, M. I., Feng, G., Brugarolas, T., Tettey, K. E. \& Lee, D. Mechanical reinforcement of nanoparticle thin films using atomic layer deposition. ACS Nano $\mathbf{5}$ 5078-5087 (2011).

30 Bédard, M. F., Munoz-Javier, A., Mueller, R., del Pino, P., Fery, A., Parak, W. J., Skirtach, A. G. \& Sukhorukov, G. B. On the mechanical stability of polymeric microcontainers functionalized with nanoparticles. Soft Matter 5, 148-155 (2009).

31 Shirman, T., Kaminker, R., Freeman, D. \& van der Boom, M. E. Halogen-bonding mediated stepwise assembly of gold nanoparticles onto planar surfaces. ACS Nano 5 , 6553-6563 (2011)

32 Such, G. K., Quinn, J. F., Quinn, A., Tjipto, E. \& Caruso, F. Assembly of ultrathin polymer multilayer films by Click chemistry. J. Am. Chem. Soc. 128, 9318-9319 (2006).

$33 \mathrm{Li}$, M., Ishihara, I., Akada, M., Liao, M., Sang, L., Hill, J. P., Krishnan, K., Ma, Y. \& Ariga, K. Electrochemical-coupling layer-by-layer (ECC-LbL) assembly. J. Am. Chem. Soc. 133, 7348-7351 (2011)

34 Li, M., Ishihara, S., Ji, Q., Ma, Y., Hill, J. P. \& Ariga, K. Electrochemical coupling layerby-layer (ECC-LbL) assembly in patterning mode. Chem. Lett. 41, 383-385 (2012).

35 Yoon, M., Kim, Y. \& Cho, J. Multifunctional colloids with optical, magnetic, and superhydrophobic properties derived from nucleophilic substitution-induced layer-bylayer assembly in organic media. ACS Nano 5, 5417-5426 (2011).

36 Osada, M., Sasaki, T., Ono, K., Kotani, Y., Ueda, S. \& Kobayashi, K. Orbital reconstruction and interface ferromagnetism in self-assembled nanosheet superlattices. ACS Nano 5, 6871-6879 (2011).

37 Ariga, K., Vinu, A., Ji, Q., Ohmori, O., Hill, J. P., Acharya, S., Koike, J. \& Shiratori, S. A layered mesoporous carbon sensor based on nanopore-filling cooperative adsorption in the liquid phase. Angew. Chem. Int. Ed. 47, 7254-7257 (2008).

$38 \mathrm{Ji}$, Q., Yoon, S. B., Hill, J. P., Vinu, A., Yu, J.-S. \& Ariga, K. Layer-by-layer films of dualpore carbon capsules with designable selectivity of gas adsorption. J. Am. Chem. Soc. 131, 4220-4221 (2009).

39 Kulkarni, D. D., Choi, I., Singamaneni, S. S. \& Tsukruk, V. V. Graphene oxidepolyelectrolyte nanomembranes. ACS Nano 4, 4667-4676 (2010).
40 Ji, Q., Honma, I., Paek, S.-M., Akada, M., Hill, J. P., Vinu, A. \& Ariga, K. Layer-by-layer films of graphene and ionic liquid for highly selective gas sensing. Angew. Chem. Int Ed. 49, 9737-9739 (2010).

41 Li, Y.-C., Mannen, S., Morgan, A. B., Chang, S. C., Yang, Y.-H., Condon, B., Jaime, C. \& Grunlan, J. C. Intumescent all-polymer multilayer nanocoating capable of extinguishing flame on fabric. Adv. Mater. 23, 3926-3931 (2011).

42 Zhu, J., Shim, B. S., Prima, M. D. \& Kotov, N. A. Transparent conductors from carbon nanotubes LBL-assembled with polymer dopant with $\pi-\pi$ electron transfer. J. Am. Chem. Soc. 133, 7450-7460 (2011).

43 Li, H., Pang, S., Wu, S., Feng, X., Müllen, K. \& Bubeck, C. Layer-by-layer assembly and UV photoreduction of graphene-polyoxometalate composite films for electronics. J. Am. Chem. Soc. 133, 9423-9429 (2011).

44 Ha, Y.-G., Emery, J. D., Bedzyk, M. J., Usta, H., Facchetti, A. \& Marks, T. J. Solutiondeposited organic-inorganic hybrid multilayer gate dielectrics. design, synthesis, microstructures, and electrical properties with thin-film transistors. J. Am. Chem. Soc. 133, 10239-10250 (2011).

45 Lee, S. W., Yabuuchi, N., Gallant, B. M., Chen, S., Kim, B.-S., Hammond, P. T. \& ShaoHorn, Y High-power lithium batteries from functionalized carbon-nanotube electrodes. Nat. Nanotechnol. 5, 531-537 (2010).

$46 \mathrm{Ma}$, Y., Zhang, Y., Wu, B., Sun, W., Li, Z. \& Sun, J Polyelectrolyte multilayer films for building energetic walking devices. Angew. Chem. Int. Ed. 50, 6254-6257 (2011).

47 Ariga, K., Ji, Q. \& Hill, J. P. Enzyme-encapsulated layer-by-layer assemblies: current status and challenges toward ultimate nanodevices. Adv. Polym. Sci. 229, 51-87 (2010).

48 Ariga, K., Lvov, Y. M., Kawakami, K., Ji, Q. \& Hill, J. P. Layer-by-layer self-assembled shells for drug delivery. Adv. Drug Deliv. Rev. 63, 762-771 (2011).

49 Ariga, K., McShane, M., Lvov, Y. M.., Ji, Q. \& Hill, J. P. Layer-by-layer assembly for drug delivery and related applications. Expert Opin. Drug Deliv. 8, 633-644 (2011).

50 Komatsu, T., Qu, X., Ihara, H., Fujihara, M., Azuma, H. \& Ikeda, H. Virus trap in human serum albumin nanotube. J. Am. Chem. Soc. 133, 3246-3248 (2011).

51 Ji, Q., Miyahara, M., Hill, J. P., Acharya, S., Vinu, A., Yoon, S. B., Yu, J.-S., Sakamoto, K. \& Ariga, K. Stimuli-free auto-modulated material release from mesoporous nanocompartment films. J. Am. Chem. Soc. 130, 2376-2377 (2008).

52 Ji, Q., Acharya, S., Hill, J. P., Vinu, A., Yoon, S. B., Yu, J.-S., Sakamoto, K. \& Ariga, K. Hierarchic nanostructure for auto-modulation of material release: mesoporous nanocompartment films. Adv. Funct. Mater. 19, 1792-1799 (2009).

53 Delcea, M., Yashchenok, A., Videnova, K., Kreft, O., Möhwald, H. \& Skirtach, A. G. Multicompartmental micro- and nanocapsules: hierarchy and applications in biosciences. Macromol. Biosci. 10, 465-474 (2010).

54 Cui, D., Jing, J., Boudou, T., Pignot-Paintrand, I., De Koker, S., De Geest, B. G., Picart, C. \& Auzély-Velty, R. Hydrophobic shell loading of biopolyelectrolyte capsules. Adv. Mater. 23, H2OO-H2O4 (2011).

55 Lee, M.-Y., Park, S.-J., Park, K., Kim, K. S., Lee, H. \& Hahn, S. K. Target-specific gene silencing of layer-by-layer assembled gold-cysteamine/siRNA/PEI/HA nanocomplex. ACS Nano 5, 6138-6147 (2011).

56 Poon, Z., Chang, D., Zhao, X. \& Hammond, P. T. Layer-by-layer nanoparticles with a pH sheddable layer for in vivo targeting of tumor hypoxia. ACS Nano 5, 4284-4292 (2011).

57 Wilson, J. T., Cui, W., Kozlovskaya, V., Kharlampieva, E., Pan, D., Qu, Z., Krishnamurthy, V. R., Mets, J., Kumar, V., Wen, J., Song, Y., Tsukruk, V. V. \& Chaikof, E. L. Cell surface engineering with polyelectrolyte multilayer thin films. J. Am. Chem. Soc. 133, 7054-7064 (2011).

58 Yang, S. H., Ko, E. H., Jung, Y. H. \& Choi, I. S. Bioinspired functionalization of silicaencapsulated yeast cells. Angew. Chem. Int. Ed. 50, 6115-6118 (2011).

59 Nishiguchi, A., Yoshida, H., Matsusaki, M. \& Akashi, M. Rapid construction of three dimensional multilayered tissues with endothelial tube networks by the cell-accumulation technique. Adv. Mater 23, 3506-3510 (2011).

60 Cohen Stuart, M. A., Huck, W. T. S., Genzer, J., Müller, M., Ober, C., Stamm, M. Sukhorukov, G. B., Szleifer, I., Tsukruk, V. V., Urban, M., Winnik, F., Zauscher, S. Luzinov, I. \& Minko, S. Emerging applications of stimuli-responsive polymer materials. Nat. Mater. 9, 101-113 (2010).

61 Delcea, M., Möhwald, M. \& Skirtach, A. G. Stimuli-responsive LbL capsules and and nanoshells for drug delivery. Adv. Drug. Deliv. Rev. 63, 630-647 (2011).

62 Skirtach, A. G., Yashchenok, A. M. \& Möhwald, H. Encapsulation, release and applications of $\mathrm{LbL}$ polyelectrolyte multilayer capsules. Chem. Commun. 47, 12736-12746 (2011).

63 Lin, Y.-H., Jiang, C., Xu, J., Lin, Z. \& Tsukruk, V. V. Sculptured layer-by-layer films. Adv. Mater. 19, 3827-3832 (2007)

64 Veerabadran, N. G., Goli, P. L., Stewart-Clark, S. S., Lvov, Y. M. \& Mills, D. K. Nanoencapsulation of stem cells within polyelectrolyte multilayer shells. Macromol. Biosci. 7, 877-882 (2007).

65 Shchepelona, O., Kozlovskaya, V., Singamaneni, S., Kharlampieva, E. \& Tsukruk, V. V. Replication of anisotropic dispersed particulates and complex continuous templates. J. Mater. Chem. 20, 6587-6603 (2010).

66 Zimnisky, D., Scevchenko, V. V. \& Tsukruk, V. V. Perforated, freely suspended layer-bylayer nanoscale membranes. Langmuir 24, 5996-6006 (2008).

67 Wu, S., Tsuruoka, T., Terabe, K., Hasegawa, T., Hill, J. P., Ariga, K. \& Aono, M. A Polymer-electrolyte-based atomic switch. Adv. Funct. Mater. 21, 93-99 (2011).

68 Hasegawa, T., Terabe, K., Tsuruoka, T. \& Aono, M. Atomic switch: atom/lon movement controlled devices for beyond von-neumann computers. Adv. Mater. 24, 252-267 (2012) 
69 Stieg, A. Z., Avizienis, A. V., Sillin, H. O., Martin-Olmos, C., Aono, M. \& Gimzewski, J. K. Emergent criticality in complex turing B-type atomic switch networks. Adv. Mater. 24, 286-293 (2012).

70 de Silva, A. P. Molecular logic gate arrays. Chem. Asian J 6, 750-766 (2011).

71 Joachim, C., Renaud, N. \& Hliwa, M. The different designs of molecule logic gates. Adv. Mater. 24, 312-317 (2012).

72 Xiang, Q., Yu, J. \& Jaroniec, M. Graphene-based semiconductor photocatalysts. Chem. Soc. Rev. 41, 782-796 (2012).

73 Tong, H., Ouyang, S., Bi, Y., Umezawa, N, Oshikiri, M. \& Ye, J. Nano-photocatalytic materials: possibilities and challenges. Adv. Mater. 24, 229-251 (2012).

74 Ratera, I. \& Veciana, J. Playing with organic radicals as building blocks for functional molecular materials. Chem. Soc. Rev 41, 303-349 (2012).

$75 \mathrm{Hu}, \mathrm{X}$. Half-metallic antiferromagnet as a prospective material for spintronics. Adv. Mater. 24, 294-298 (2012).

76 Wang, Z. L. Self-powered nanosensors and nanosystems. Adv. Mater. 24, 280-285 (2012).

77 Ariga, K., Mori, T. \& Hill, J. P. Control of nano/molecular systems by application of macroscopic mechanical stimul. Chem. Sci 2, 195-203 (2011).

78 Ariga, K., Mori, T. \& Hill, J. P. Mechanical control of nanomaterials and nanosystems. Adv. Mater. 24, 158-176 (2012).

\section{(c)} SERVE

This work is licensed under the Creative Commons Attribution-NonCommercial-Share Alike 3.0 Unported License. To view a copy of this license, visit http://creativecommons. org/licenses/by-nc-sa/3.0/

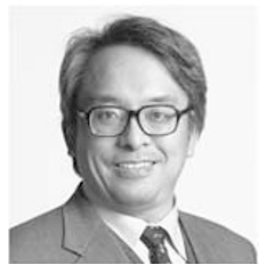

Katsuhiko Ariga received his BE, ME. and PhD degrees from the Tokyo Institute of Technology (TIT). He was an Assistant Professor at TIT, and worked as a postdoctoral fellow at the University of Texas at Austin, USA and then served as a group leader in the Supermolecules Project at the Japan Science and Technology (JST) agency. Thereafter, he worked as an Associate Professor at the Nara Institute of Science and Technology, and then he got involved with the Exploratory Research for Gothic Advanced Technology Nanospace Project at JST. In January 2004, he moved to National Institute for Materials Science. He has been also appointed as a Principal Investigator of World Premier International Research Center for Materials. His major interests are the fabrication of novel functional nanostructures based on molecular recognition and self-assembly including Langmuir-Blodgett films, layer-by-layer films and mesoporous materials. In 2010, he was awarded as a Nice-Step Researcher.
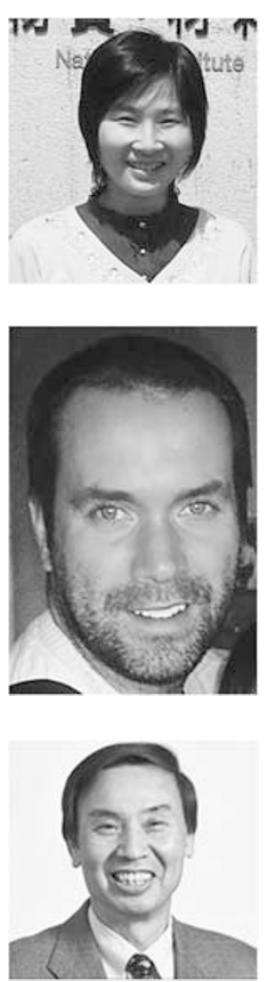

Professor Yoshio Bando is a Fellow of the National Institute for Materials Science (NIMS) and the Chief Operating Officer, International Center for Materials Nanoarchitectonics, NIMS. Since 2008, he has been Adjunct Professor at both The University of Tokyo and Waseda University. In 1975, he received a PhD degree at the Osaka University. His current research concentrates on the synthesis and properties of novel inorganic nanotubes/nanowires and transmission electron microscopy analysis. He has received a number of awards including the Tsukuba Prize (2005), and he has been selected as an ISI highly cited researcher in Materials Science. He is a Fellow of The American Ceramic Society, and he is also serving as the Editor-in-chief of Journal of Electron Microscopy (since 2009).

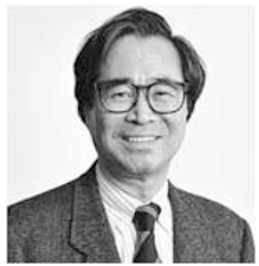

Masakazu Aono received a PhD degree in Metallurgy from the University of Tokyo in 1972. Subsequently, he became a Research Staff Member of the National Institute for Research in Inorganic Materials. In 1986, he moved to RIKEN as a Chief Scientist and maintained a laboratory there until 2002. From 1996 to 2002, he was also a Professor at Osaka University. In 2002, he joined the National Institute for Materials Science as the Director General of the Nanomaterials Laboratory. In 2007, he assumed the role of the Director General of the International Center for Materials Nanoarchitectonics. He has made, and continues to make, pioneering contributions in the fields of surface science, nanoscience and nanotechnology with novel analytical instrumentation and advanced technologies. 